supports guideline developers adjusting implementation strategies and improving updating.

Description of Best Practice We developed a prototype, which uses input based on disease (TNM, stadium) and patient characteristics (co-morbidity, e.g.). First, recommendations were formulated as computer interpretable recommendations using IF... THEN rules. Second, the application assembled all information and combined them with alerts, namely contraindications and side effects, finally leading to treatment advice. We found that CDS is a viable way of assisting doctors and patients. Treatment advice is better suited to both evidence based recommendations and specific patient characteristics. Insight into why a certain choice is made improves confidence in the suggested treatment and compliance. Also, more gaps in knowledge were found and trial participation was improved.

Lessons for Guideline Developers/Users CDS: Can provide insight into the use of guidelines. For example, when a recommendation isn't followed, possible efforts in implementation (recommendation is not/poorly implemented) or update (recommendation is outdated) are needed. Rewriting recommendations increased consistent language used in guidelines, which include easy reuse of data between professionals, hospitals and Cancer Registry Updating guidelines is expensive and time consuming. The doctors (and patients) ability to respond to existing recommendations supports faster, more efficient and cheaper modular updates.

\section{P127 COMPREHENSIVE MODEL FOR IMPLEMENTATION OF GUIDELINES FOR DISEASE PREVENTION}

M Elgstrand, I Carlsson, A Månsdotter, R Sorsa, C Strååt. The National Board of Health and Welfare, Stockholm, Sweden

\section{0:1136/bmjqs-2013-002293.173}

Background The National Board of Health and Welfare (NBHW) has developed guidelines for disease prevention, which include interventions to reduce smoking, hazardous use of alcohol, insufficient physical activity and unhealthy diet.

Context To support the implementation of these guidelines, the Government has commissioned the NBHW to disseminate knowledge, create Web-based training, and ensure data access and methodological development.

Description of Best Practice Implementation of guidelines for disease prevention deserves a comprehensive approach, since it involves most health care settings and professions. The NBHW coordinates national stakeholders, including decision makers at the regional level, in several networks. Organisations for health professionals such as doctors, nurses, physiotherapists and dieticians, receive support to disseminate knowledge of the guidelines among their members. Other ways of disseminating knowledge include information on the NBHW website and leaflets for patients, managers and professionals. The NBHW will also develop a Webb-based training for health care professionals, covering the methods recommended in the guidelines. To support improvements in methods for disease prevention, research groups have been awarded financial support, for example to study how P4P can be used to improve implementation of guidelines and how to support patients with special needs. Furthermore, the NBHW supports harmonisation of registration and reporting on data. The NBHW will also publish a national assessment and evaluation, in order to identify differences between regions regarding organisational factors, processes, clinical outcomes and costs.
Lessons Learned We will share our experiences of a comprehensive approach, targeting decision makers, health care professionals and patients, and discuss challenges when translating guidelines into health care.

\section{P129 ESTIMATING SERVICE CAPACITY FOR COMMISSIONING AN ANTICOAGULATION SERVICE IN LINE WITH NICE GUIDANCE IN THE NHS, ENGLAND}

D Moran, P Griffiths, V Moore. NICE, Manchester, UK

\section{0:1136/bmjqs-2013-002293.174}

Background Improving quality, patient outcomes and cost effectiveness is an assumed aim of health and social care commissioning. A key aspect for commissioners in the planning phase of the commissioning cycle for an anticoagulation service, in line with NICE guidance, is the ability to estimate the level of service that will be required in order to appropriately commission or decommission services.

Objectives Establish the level of integration of information, alongside clinical and management knowledge, required to successfully calculate appropriate service levels when commissioning or decommissioning an anticoagulation services in the NHS.

Methods A critical appraisal of clinical research studies, epidemiological data, NHS activity data and other information was carried out in combination with clinical and NHS management oversight to inform an estimate of service levels for an anticoagulation service. A systematic literature search of 3 electronic databases, Medline, Embase and Cochrane was carried out. Routinely collected activity data was reviewed through a sample of GP practice systems for primary care information and hospital episode statistics for secondary care information. Healthcare professionals and commissioners with a specialty or interest in an anticoagulation service were consulted.

Results Interim results suggest integration of multiple information sources in combination with clinical and management knowledge produces more robust estimates of service levels for an anticoagulation service.

Discussion The accuracy, and therefore the utility of estimates of service levels for an anticoagulation service will be improved by information linkage, and by using intelligence from multiple sources. This approach could be applied to estimating service levels for other commissioned services.

\section{P132 QUALITY OF GUIDELINES DEVELOPED BY THE WORLD HEALTH ORGANIZATION: PRELIMINARY RESULTS}

${ }^{1}$ B Burda, ${ }^{2}$ A Chambers. ' Kaiser Permanente Center for Health Research, Portland, USA; ${ }^{2}$ Pacific University, Forest Grove, USA

10:1136/bmjqs-2013-002293.175

Background The World Health Organization (WHO) annually publishes hundreds of guidelines. Its guideline development process, however, is often criticised even after the implementation of a Guideline Review Committee (GRC) that ensure guidelines are developed using the highest methodological quality, transparent and evidence-based processes.

Objectives To quality rate a cohort of GRC-approved WHO guidelines using the Appraisal of Guidelines for Research and Evaluation (AGREE) II tool. 